\title{
Set-valued estimation of switching linear system: an application to an automotive throttle valve
}

\author{
Elena Podivilova ${ }^{1 *}$, Alessandro N. Vargas ${ }^{2}$, Vladimir Shiryaev $^{1}$, Leonardo Acho ${ }^{3}$ \\ ${ }^{1}$ Control Systems Department, South Ural State University (National Research University), 76, Lenin \\ prospekt, Chelyabinsk, Russia, \\ ${ }^{2}$ Electrotechnical Department, Universidade Tecnológica Federal do Paraná, UTFPR, Av. Alberto \\ Carazzai 1640, 86300-000 Cornelio Procópio-PR, Brazil, \\ ${ }^{3}$ Departament de Matemàtica Aplicada III, Universitat Politécnica de Catalunya, Comte d'Urgell, \\ 187, 08036 Barcelona, Spain
}

\begin{abstract}
SUMMARY
This paper introduces a polyhedral approximation algorithm for set-valued estimation of switching linear systems. The algorithm generates set-valued estimates for any possible sequence of switching parameters, under the assumption that the system has unknown but bounded disturbances and measurement noises. Our algorithm has practical implications; namely, setvalued estimates were generated for the position and electrical current of a real-time automotive electronic throttle valve, and the corresponding experimental data demonstrate the practical benefits of our approach. Copyright (c) 2015 John Wiley \& Sons, Ltd.
\end{abstract}

Received ...

KEY WORDS: Switching systems; set-valued estimation; automotive applications

\section{INTRODUCTION}

Switching linear systems comprise a class of dynamical systems that have solution driven by switching signals. Much research in recent years has focused on the study of such systems, see for instance the monograph [1] and the papers [2, 3, 4], for a brief account. The case in which the switching signal is modified so as to stabilize the system has also attracted attention, see [5], [6], [4], and [7], and the references therein. Although the study of stability of switching linear systems has reached a relatively mature level, a little attention has been paid to the estimation problem.

As an attempt to estimate the switching system state, we could use Bayesian filters [8] and unscented Kalman filters [9, 10]. A drawback of these approaches is that they rely on probabilistic methods, so that the estimated value can be far away from the real value. That discrepancy in the estimation may lead the process to reach values that can damage the underlying equipments.

On the contrary, set-valued estimation generates sets that assuredly contain the real value of the system state - set-valued estimation thus seems to be a reasonable technique to be used in practice. Note that the set-valued estimation allows us to check whether the system state may assume undesirable values in practice. This finding has implications for applications, as illustrated in Section 3.

${ }^{*}$ Correspondence to: Control Systems Department, South Ural State University (National Research

University), 76, Lenin prospekt, Chelyabinsk, Russia. E-mail: podivilovaeo@susu.ac.ru 
The papers [11] and [12] suggest to perform set-valued estimation for each subsystem and next joining all of the corresponding sets into a single set; they consider feasible set outer approximation for linear systems with some canonical forms, like ellipsoids [13, 14, 15, 16], parallelotopes $[17,18,19]$, and zonotopes [20]. These methods rely on computing set-valued estimation through Minkowski sum and set intersection, accounted individually for each subsystem.

In contrast, we construct set-valued estimates without Minkowski sum and intersection by using polyhedral approximation. The polyhedral approximation has advantage of not only allowing a polyhedra of any shape, but also decreasing the size of the corresponding feasible set, thus mitigating the loss of accuracy. This sets the main theoretical contribution of this paper.

Actually, our algorithm can construct polyhedrons of any shape so as to contain the system state - the algorithm requires the system equations and some linear inequalities describing disturbance and noise sets [21]. The solution of the algorithm is obtained by solving linear programming problems.

Our theoretical contribution has practical implications. Indeed, real-time experiments were carried out in a laboratory testbed to generate set-valued estimations for the position and electrical current of an automotive electronic throttle device, a device largely used by the automotive industry.

The position of the throttle device is measured by a sensor subject to measurement errors. Considering only information from this sensor, we used our set-valued method to generate estimations for both the position and current of the throttle. Thus, the paper contributes not only for the theory of set-valued estimation but also for real-time applications.

\section{ALGORITHM FOR SET-VALUED ESTIMATION}

The discrete-time switching linear system under investigation is driven by the following equations:

$$
\begin{aligned}
& x_{k+1}=A_{\sigma(k)} x_{k}+B_{\sigma(k)} u_{k}+F_{\sigma(k)} w_{k} \\
& y_{k+1}=C_{\sigma(k)} x_{k+1}+D_{\sigma(k)} v_{k+1}, \quad k=0,1, \ldots, N,
\end{aligned}
$$

where $x_{k} \in \mathbb{R}^{n_{x}}$ is the system state vector, $u_{k} \in \mathbb{R}^{n_{u}}$ is the given input vector, $w_{k} \in \mathbb{R}^{n_{w}}$ is the disturbance vector, $y_{k} \in \mathbb{R}^{n_{y}}$ is the measurement vector, and $v_{k} \in \mathbb{R}^{n_{v}}$ is the measurement noise vector. For each $k \geq 0$, the matrix $A_{\sigma(k)} \in \mathbb{R}^{n_{x} \times n_{x}}$ belongs to a given set $\left\{A_{1}, \ldots, A_{M}\right\}$, so that $A_{\sigma(k)}=A_{m}$ whenever $\sigma(k)=m \in\{1, \ldots, M\}$; the same applies for the matrices $B_{\sigma(k)}, F_{\sigma(k)}, C_{\sigma(k)}$ and $D_{\sigma(k)}$. The initial state $x_{0} \in \mathbb{R}^{n_{x}}$, disturbances $w_{k} \in \mathbb{R}^{n_{w}}$, and noises $v_{k} \in \mathbb{R}^{n_{v}}$ are defined by polyhedral sets, i.e.,

$$
\begin{gathered}
x_{0} \in \bar{X}_{0}=\left\{x_{0} \in \mathbb{R}^{n_{x}} \mid H_{x_{0}} x_{0} \leq b_{x_{0}}\right\}, \\
w_{k} \in W=\left\{w \in \mathbb{R}^{n_{w}} \mid H_{w} w_{k} \leq b_{w}\right\}, \\
v_{k} \in V=\left\{v \in \mathbb{R}^{n_{v}} \mid H_{v} v_{k} \leq b_{v}\right\},
\end{gathered}
$$

where $H_{x_{0}}, H_{w}$, and $H_{v}$ are given matrices of appropriate dimensions.

The set-valued estimation technique involves a construction of feasible sets $\bar{X}_{k}$, which are guaranteed to contain all the possible values of the system state, i.e., $x_{k} \in \bar{X}_{k}$ for each $k \geq 0$ [15]. Such sets are constructed by using the following equations, for each $k \geq 0$ :

$$
\begin{aligned}
X_{k+1 \backslash k} & =A_{\sigma(k)} \bar{X}_{k}+B_{\sigma(k)} u_{k}+F_{\sigma(k)} W, \\
X\left[y_{k+1}\right] & =\left\{x \in \mathbb{R}^{n_{x}} \mid C_{\sigma(k)} x+D_{\sigma(k)} v=y_{k+1}, \forall v \in V\right\}, \\
\bar{X}_{k+1} & =X_{k+1 \backslash k} \cap X\left[y_{k+1}\right] .
\end{aligned}
$$

Remark 1

All operations in (3) are set operations, that is, linear transformation of sets, Minkowski 
sum of sets, and set intersection. The reachable set $X_{k+1 \backslash k}$ denotes set of states accessible from previous feasible set $\bar{X}_{k}$.

Given (1) and (2), each new measurement $y_{k+1}$ allows us to compute the corresponding state set $X\left[y_{k+1}\right]$. Both sets $X_{k+1 \backslash k}$ and $X\left[y_{k+1}\right]$ contain the real state of the system; and the intersection of both sets generate the feasible set $\bar{X}_{k+1}$ (see (3)).

Computing the feasible set $\bar{X}_{k+1}$ is a difficult task. To overcome such drawback, we present the next polyhedral approximation algorithm.

\section{Algorithm}

Step 1. Set the iterations counter at $k=0$.

Step 2. At the sample time $k$, the input $u_{k}$ and measurement $y_{k+1}$ are given, but the terms $x_{k+1}, x_{k}, w_{k}$ and $v_{k+1}$ are unknown. After rearranging the unknown parameters, we have from (1) that

$$
\left[\begin{array}{cccc}
I & -A_{m} & -F_{m} & 0 \\
C_{m} & 0 & 0 & D_{m}
\end{array}\right]\left[\begin{array}{c}
x_{k+1} \\
x_{k} \\
w_{k} \\
v_{k+1}
\end{array}\right]=\left[\begin{array}{c}
B_{m} u_{k} \\
y_{k+1}
\end{array}\right], \quad m=1, \ldots, M .
$$

Step 3. From (2), we obtain the following set of restrictions:

$$
\left[\begin{array}{cccc}
0 & H_{x_{k}} & 0 & 0 \\
0 & 0 & H_{w} & 0 \\
0 & 0 & 0 & H_{v}
\end{array}\right]\left[\begin{array}{c}
x_{k+1} \\
x_{k} \\
w_{k} \\
v_{k+1}
\end{array}\right] \leq\left[\begin{array}{c}
b_{x_{k}} \\
b_{w} \\
b_{v}
\end{array}\right]
$$

Step 4. The systems in (4) and (5) implicitly describe the feasible set $\bar{X}_{k+1}$. It is necessary to present the explicit description. To this end, we calculate upper polyhedral approximation $X_{k+1}$ of the feasible set $\bar{X}_{k+1} \subseteq X_{k+1}$ as a linear inequality system:

$$
X_{k+1}=\left\{x_{k+1} \mid H_{x_{k+1}} x_{k+1} \leq b_{x_{k+1}}\right\} .
$$

Matrix $H_{x_{k+1}}$ is given, that is, it is chosen according to the directions in which the values of the system state are the mostly significant. For instance, when we need bounds of coordinates for the vector $x_{k}$, the matrix $H_{x_{k+1}}$ can describe a parallelepiped, i.e., $H_{x_{k+1}} \equiv\left[\begin{array}{c}I \\ -I\end{array}\right]$, where $I$ denotes the identity matrix of dimension $n_{x} \times n_{x}$. To get the values of $b_{x_{k+1}}$, it is necessary to solve a row of linear programming problems. For each $m \in\{1, \ldots, M\}$ and for each $h_{i}(i$-th row of matrix $H_{x_{k+1}}$ ), the following problems are solved:

$$
x^{*}=\underset{x_{k+1}}{\arg \max }\left\langle h_{i}, x_{k+1}\right\rangle \text { subject to (4) and (5), }
$$

where $\langle\cdot, \cdot\rangle$ is a scalar product of vectors. Then

$$
b_{x_{k+1}, m}(i)=\left\langle h_{i}, x^{*}\right\rangle,
$$

The value of $b_{x_{k+1}}$ can be calculated the following way:

$$
b_{x_{k+1}}(i)=\max _{m \in\{1, \ldots, M\}} b_{x_{k+1, m}}(i) .
$$

Step 5. If $k=N$, then the algorithm stops; otherwise set $k=k+1$ and go to Step 2 .

Remark 2

If some information about the switching rule $\sigma(k)$ is known in advance, then this information can be used to alleviate the computational burden of the algorithm. For instance, instead of computing the sets for every $m \in\{1, \ldots, M\}$, we can compute subsets $S \subset\{1, \ldots, M\}$ driven by the rule $\sigma(k)$. 


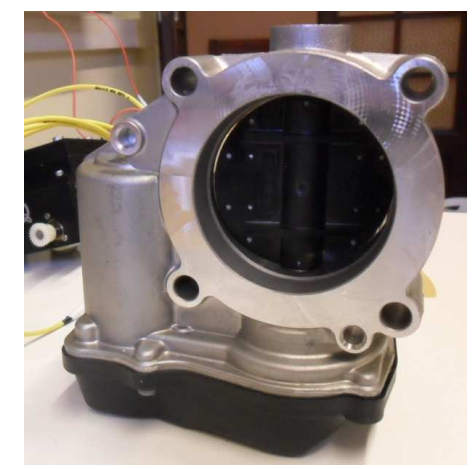

Figure 1. Automotive electronic throttle device used in the experiments described in Section 3.

Since the sets $W, V$ and $\bar{X}_{0}$ are closed and bounded, the resulting feasible sets $\bar{X}_{k}$, $k \geq 0$, and their upper approximations $X_{k}, k \geq 0$, are also closed and bounded. Besides, in most of the applications, the autonomous switching linear system $x_{k+1}=A_{\sigma(k)} x_{k}$ is globally asymptotically stable; thus the influence of the disturbance set $W$ in the feasible set $X_{k}$ diminishes as the time evolves, and $X_{k}$ remains bounded when $k \rightarrow \infty$. Notice that the accuracy of set-valued estimate $X_{k}$ depends not only on the size of the sets $W, V$ and $\bar{X}_{0}$, but also on the disturbance and noise realization.

\section{SET-VALUED ESTIMATION FOR AN AUTOMOTIVE ELECTRONIC THROTTLE VALVE}

This section presents an application of the set-valued algorithm in Steps 1-5. Actually, the algorithm of Steps 1-5 was used in practice to estimate both position and electrical current consumed by an automotive throttle device, as detailed next.

The automotive electronic throttle body is a device largely used by the automotive industry to regulate the power generated by spark-ignition combustion engines $[22,23$, $24,25]$. Applying a voltage in the throttle's input terminals makes the internal valve spin accordingly, and a sensor of position measures the angular movement made by the valve. In the throttle's model, the voltage represents the input and the angular position of the valve represents the output.

Two points motivated us to perform the experiments. First, the sensor used to measure the position was modified to produce an error of 9/256 Volts (quantization error of 8 bits to represent 9 Volts). Second, there is no sensor for measuring the electrical current consumed by the throttle; however, the peak values in the current can damage the involved electronic circuitry. Because our algorithm can assure the minimum and maximum values taken by the electrical current, even under quantization errors, our algorithm can detect extreme values of electrical current, as detailed next.

\subsection{Modelling and identification}

The laboratory testbed used to perform the experiments was equipped with the following devices: a unity of Quanser Q4 Real-Time Control Board that implemented the data communication with a computer; a unity of Quanser UPM180-25-B-PWM Power Amplifier that supplied the voltage and electrical current consumed by the equipments; and a unity of the automotive electronic throttle body made up by Continental Siemens VDO, Model A2C59511705, P.N. 06F133062J (Fig. 1). For more details about the setup of the laboratory, see $[26]$.

The literature suggests a three-dimensional system to represent the automotive electronic throttle body [27, 25]: (i) the angular position of the throttle valve; (ii) the angular velocity of 
the throttle valve; and (iii) the electrical current consumed by the throttle's internal motor. Because the throttle is equipped with just one sensor to measure the angular position of the valve, applying numerical derivative to the angular position yields the angular velocity. However, there is no sensor to measure the electrical current consumed by the throttle, the third element of the system state.

To measure the electrical current, one must use an external circuitry, completely detached from the throttle structure, which can be costly and inconvenient. Interestingly, our algorithm in Steps 1-5 can be used to estimate intervals of the electrical current, a clear advantage of our approach for the practical point of view.

Although it has been known that the throttle is a nonlinear device [22, 25, 28], we decided to represent it as a piecewise linear system, an idea borrowed from [29] and [30]. The traditional nonlinear continuous-time model of the throttle can be written as (e.g., [31, Eq. (6)],[25, Eq. (6)], [27, Eq. (8)]),

$$
\begin{aligned}
\dot{\theta}_{t} & =a_{12} \omega_{t}, \\
\dot{\omega}_{t} & =a_{21} \omega_{t}+a_{22} \theta_{t}+a_{23} y_{t}+c+f\left(\theta_{t}, \omega_{t}\right), \\
\dot{y}_{t} & =a_{32} \omega_{t}+a_{33} y_{t}+b u_{t},
\end{aligned}
$$

where $\theta_{t}$ denotes the angular position, $\omega_{t}$ denotes the angular velocity, and $y_{t}$ denotes the electrical current; here, $f$ is a nonlinear function, and the constants $a_{12}, \ldots, a_{33}, c$, are fixed.

Setting $x_{t} \equiv\left[\begin{array}{lll}\theta_{t} & \omega_{t} & y_{t}\end{array}\right]^{\prime} \in \mathbb{R}^{3}$, neglecting the nonlinear term $f\left(\theta, \omega_{t}\right)$, and applying the usual Euler discretization in the resulting model, we obtain the discrete-time piecewise linear system

$$
\begin{aligned}
x_{k+1} & =\left[\begin{array}{ccc}
1 & a_{12}^{(m)} & 0 \\
a_{21}^{(m)} & a_{22}^{(m)} & a_{23}^{(m)} \\
0 & a_{32}^{(m)} & a_{33}^{(m)}
\end{array}\right] x_{k}+\left[\begin{array}{c}
0 \\
0 \\
b^{(m)}
\end{array}\right] u_{k}+w_{k}+\left[\begin{array}{c}
0 \\
c^{(m)} \\
0
\end{array}\right], \\
y_{k} & =\left[\begin{array}{lll}
1 & 0 & 0
\end{array}\right] x_{k}+v_{k}, \quad x_{1, k} \in \Gamma_{m}, \quad m=1, \ldots, 3,
\end{aligned}
$$

where the values of $a_{12}^{(m)}, \ldots, a_{33}^{(m)}, b^{(m)}, c^{(m)}, m=1, \ldots, 3$, are available in Table I. Note that $x_{k} \equiv\left[\begin{array}{lll}x_{1, k} & x_{2, k} & x_{3, k}\end{array}\right]^{\prime} \in \mathbb{R}^{3}$ stands for the system state with $x_{1, k}$ as position (rescaled by a factor of 0.1 hereafter), $x_{2, k}$ as angular velocity, and $x_{3, k}$ as electrical current.

The sets $\Gamma_{1}, \Gamma_{2}$, and $\Gamma_{3}$ denote the partition for the position, so that $x_{1, k}$ takes values from the sets $\Gamma_{1}=[0,0.8], \Gamma_{2}=(0.8,1.6]$, and $\Gamma_{3}=(1.6,9]$. Additionally, the $m$-th mode is active in (10) when $x_{1, k}$ belongs to $\Gamma_{m}$.

To identify the values presented in Table I, persistent excitation signals were applied in practice in the input $u_{k}$, and the corresponding data for $x_{k}$ were recorded. In such a procedure, we carefully selected and used a total of 80,000 points. By minimizing the mean square error between the simulated and real-time data, we obtained the values shown in Table I. Afterwards, the obtained model was compared with the remaining part of the real-time data for verification and validation.

Table I. Parameters for the piecewise linear system modelling an automotive throttle body.

\begin{tabular}{cccc}
\hline Parameter & $m=1$ & $m=2$ & $m=3$ \\
\hline$a_{12}^{(m)}$ & -3.4483 & -2.8017 & -0.12381 \\
$a_{21}^{(m)}$ & 0.00012 & 0.002 & 0.0938 \\
$a_{22}^{(m)}$ & 0.9358 & -0.1677 & -0.6864 \\
$a_{23}^{(m)}$ & -0.0006 & -0.0221 & -1.9807 \\
$a_{32}^{(m)}$ & -0.3913 & -1.193 & -0.0128 \\
$a_{33}^{(m)}$ & 0.94486 & 0.9494 & 0.9394 \\
$b^{(m)}$ & 0.07179 & 0.04182 & 0.02607 \\
$c^{(m)}$ & 0.0001 & 0.0037 & 0.1728 \\
\hline
\end{tabular}




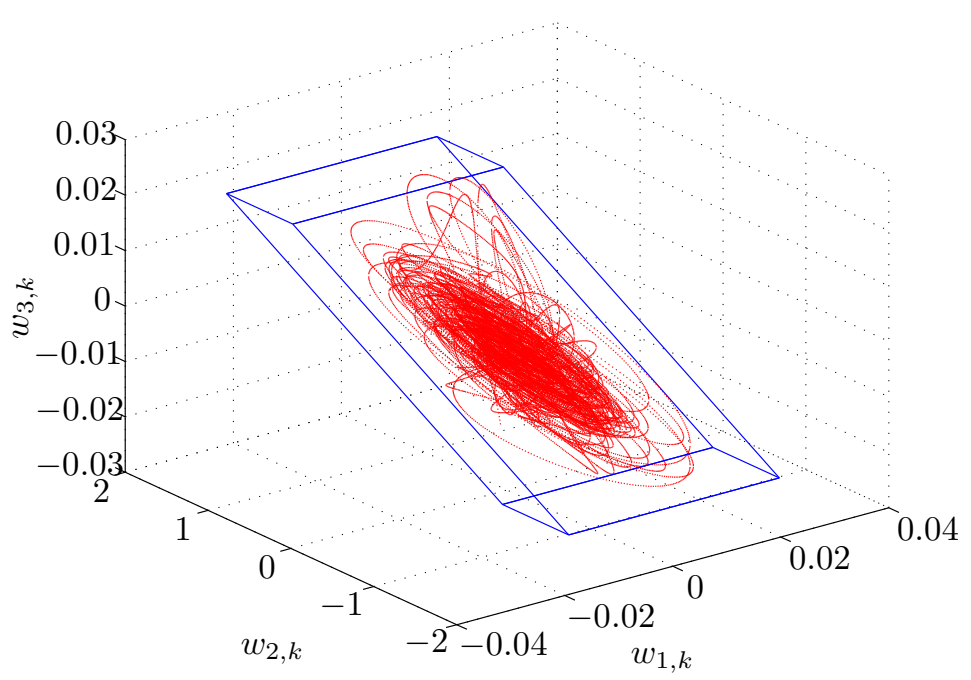

Figure 2. Error in the model that represents an automotive electronic throttle valve. The trajectory of the error is bounded by a parallelepiped, which in turn represents the disturbance set $W$ in (10).

Next, the data acquisition board was configured to work under 8-bit resolution in order to emulate a sensor of position with quantization error of 8 bits. For this reason the error disturbance $v_{k}$ in (10) was defined by the set $V$, as in (2), with vectors

$$
H_{v}=\left[\begin{array}{c}
1 \\
-1
\end{array}\right], \quad b_{v}=\left[\begin{array}{l}
9 / 256 \\
9 / 256
\end{array}\right]
$$

The set $W$ that defines the disturbance the term $w_{k}$ in (10) was characterized as follows. A total of 40,000 new points were collected in the laboratory; we used these points to calculate the error $e_{k}=x_{k}-\tilde{x}_{k}$, where $x_{k}$ denotes the solution of (10) with $w_{k} \equiv 0$ and $\tilde{x}_{k}$ denotes the corresponding point measured in practice. The error points generated the trajectory of $w_{k}$; we suggested a form of parallelepiped for $W$ in (2) in order to obtain $w_{k} \in W$, see Fig. 2 .

The matrices of $W$ are

$$
H_{w}=\left[\begin{array}{ccc}
-0.0408 & 0 & -0.02 \\
0 & 0.001989 & -0.0663 \\
0.0408 & 0 & 0.02 \\
0 & -0.001989 & 0.0663 \\
0 & 0 & 1 \\
0 & 0 & -1
\end{array}\right], \quad b_{w}=\left[\begin{array}{c}
0.0008456 \\
0.0010608 \\
0.0007456 \\
0.0005704 \\
0.024 \\
0.028
\end{array}\right] .
$$

\subsection{Experimental results for set-valued estimation}

The code for computing the algorithm in Steps 1-5 was written in Matlab R2010a and tested on a computer with $2.20 \mathrm{GHz}$ CPU Intel(R) Core(TM) i7-4702MQ and 8Gb RAM. In this computer, the time necessary to compute each estimation set was close to 52 milliseconds.

The algorithm of Steps 1-5 was used to estimate the sets containing the real-time state of the automotive electronic throttle device. In the practical experiments with the throttle, both the voltage input $u_{k}$ and the measured position $y_{k}$ were used to feed the algorithm of Steps 1-5. The initial state was kept fixed at $x_{0}=\left[\begin{array}{lll}0 & 0 & 0\end{array}\right]^{\prime}$. To simplify the 


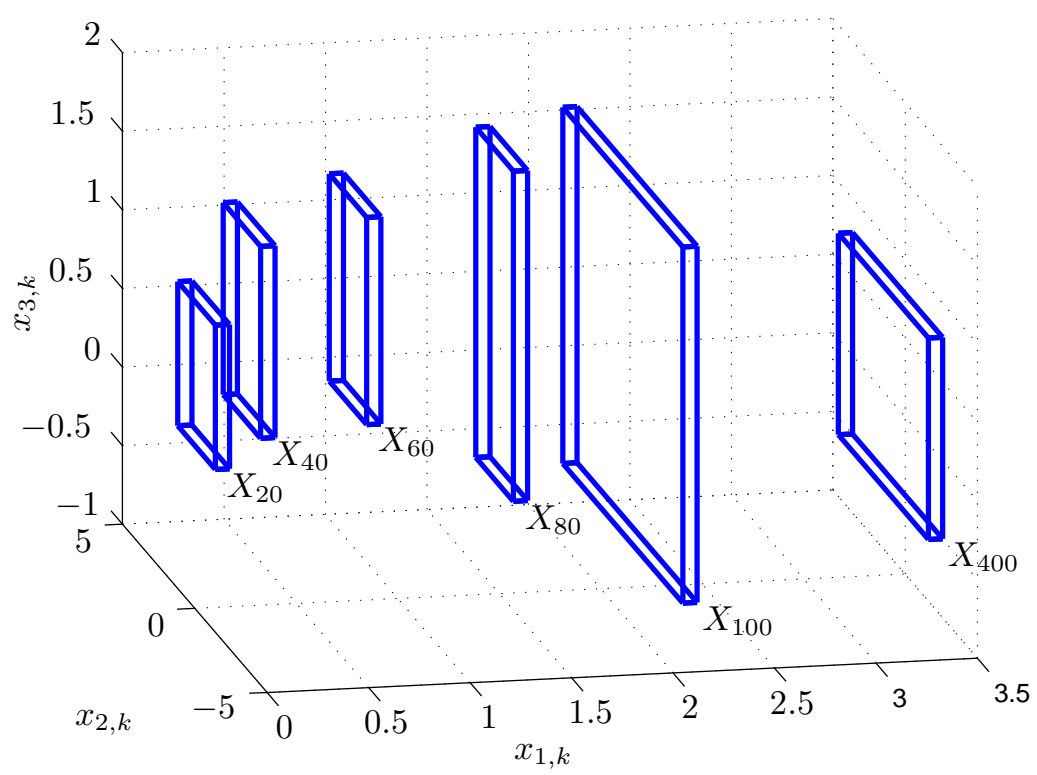

Figure 3. Examples of set-valued estimates $X_{k}$ for some iterations $(k=20,40, \ldots, 100,400)$, where $x_{1, k}, x_{2, k}, x_{3, k}$ represent position, angular velocity and electrical current correspondingly.

visualization of the results, the feasible sets were approximated with parallelepipeds, i.e., we set $H_{x_{k+1}}=\left[\begin{array}{c}I_{3 \times 3} \\ -I_{3 \times 3}\end{array}\right]$ for all $k>0$.

More than one hundred thousand points were collected and stored. Afterwards, the collected data were applied into the algorithm of Steps 1-5.

Fig. 3 illustrates the feasible set approximations for some distinct iteration values.

Fig. 4 illustrates the estimated values for the first 2500 points. As expected, all the practical values laid within the corresponding estimated sets. As can be seen, even under quantization error, the estimated bounds for the position are quite narrow.

Note also in Fig. 4 that the electrical current of the throttle kept within the limits calculated by the set-valued estimation of Steps 1-5; this finding illustrates the usefulness of our approach for detecting extreme values of the current consumed by the throttle.

The experimental data suggest that our algorithm obey the constraints in (5) and (4).

In summary, the experimental data confirmed the correctness of the estimation procedure, a clear indication that our set-valued estimation technique can be useful in practice.

\section{CONCLUDING REMARKS}

We have presented an algorithm for set-valued estimation of switching linear systems. The switching system accounts bounded disturbances for the input and output. An advantage of our approach is that the system state assuredly keeps within the estimated set.

Our findings have practical implications. In reality, practical experiments were carried out in a laboratory testbed to generate set-valued estimation for the position and electrical current of an automotive electronic throttle device. The position was measured through a sensor with quantization errors; using such measurement, we produced set-valued estimation for the electrical current consumed by the throttle. The experiments illustrate the potential benefits of our algorithm for applications. 

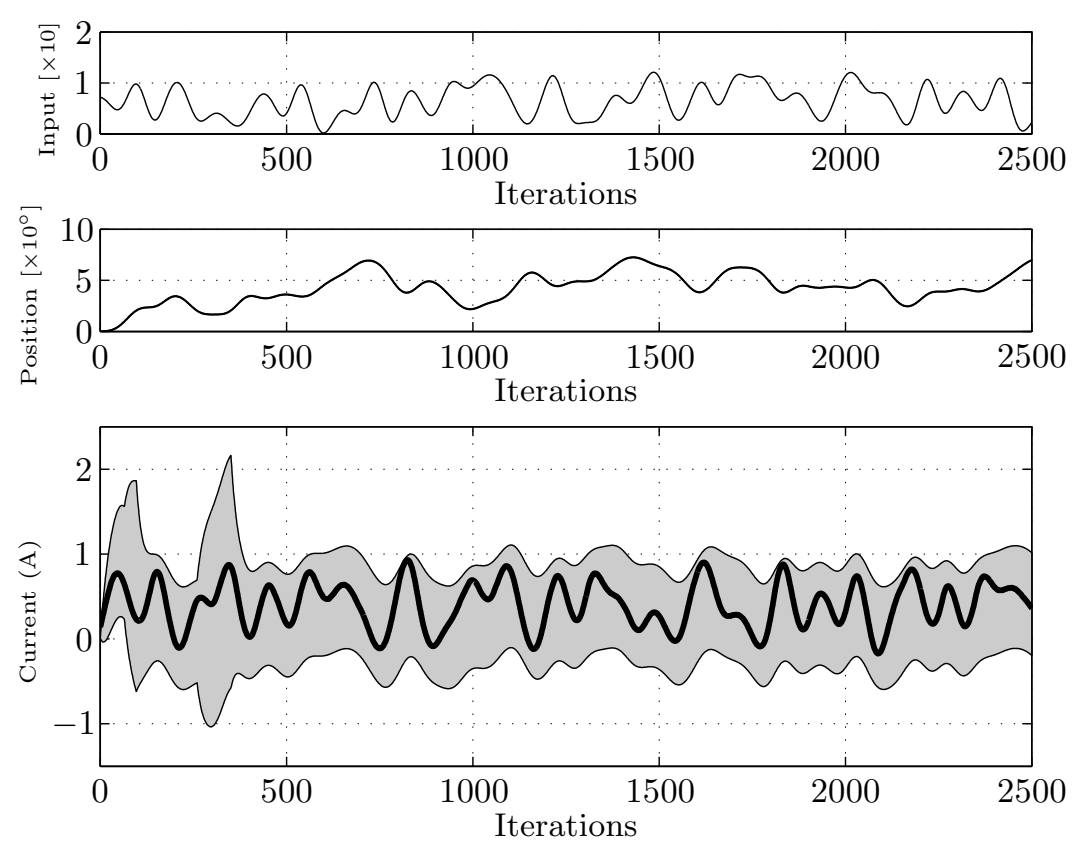

Figure 4. Experimental data for an automotive electronic throttle device. Real-time values from the input $u_{k}$ (Volts) and output $y_{k}$ (Angular degree) were used to generate the sets that estimated the position of the throttle $x_{1, k}$ (Angular degree) and the electrical current $x_{3, k}(\mathrm{~A})$. The bold black lines correspond to real-time data, and these lie within tubes that represent the estimated sets (sampling time of $1 \mathrm{~ms}$ for each iteration).

\section{ACKNOWLEDGEMENTS}

Research supported in part by the Spanish Ministry of Economy and Competitiveness through the research project DPI2011-28033-C03-01 and through grant DPI201232375/FEDER and by the Brazilian agencies CNPq Grant 248470/2013-2; and CAPES Grant Programa PVE 88881.030423/2013-01.

\section{REFERENCES}

1. D. Liberzon, Switching in Systems and Control, Birkhäuser, Boston, USA, 2003.

2. R. Shorten, F. Wirth, O. Mason, K. Wulff, C. King, Stability criteria for switched and hybrid systems, SIAM Rev. 49 (2007) 545-592.

3. M. I. Krastanov, V. M. Veliov, On the controllability of switching linear systems, Automatica 41 (4) (2005) 663-668.

4. H. Lin, P. J. Antsaklis, Stability and stabilizability of switched linear systems: A survey of recent results, IEEE Trans. Automatic Control 54 (2) (2009) 308-322.

5. F. Blanchini, C. Savorgnan, Stabilizability of switched linear systems does not imply the existence of convex Lyapunov functions, Automatica 44 (4) (2008) 1166-1170.

6. J. C. Geromel, P. Colaneri, P. Bolzern, Dynamic output feedback control of switched linear systems, Automatic Control, IEEE Trans. 53 (3) (2008) 720-733.

7. J. P. Hespanha, Uniform stability of switched linear systems: extensions of LaSalle's invariance principle, Automatic Control, IEEE Trans. 49 (4) (2004) 470-482.

8. M. Arulampalam, S. Maskell, N. Gordon, T. Clapp, A tutorial on particle filters for online nonlinear/non-Gaussian Bayesian tracking, Signal Processing, IEEE Trans. 50 (2) (2002) 174-188.

9. R. Kandepu, B. Foss, L. Imsland, Applying the unscented Kalman filter for nonlinear state estimation, Journal of Process Control 18 (7-8) (2008) 753-768.

10. H. M. Menegaz, J. Y. Ishihara, G. A. Borges, A. N. Vargas, A systematization of the unscented Kalman filter theory (in press), IEEE Trans. Automat. Control Doi:10.1109/TAC.2015.2404511.

11. M. Baglietto, G. Battistelli, L. Scardovi, Active mode observation of switching systems based on setvalued estimation of the continuous state, International Journal of Robust and Nonlinear Control 
19 (2009) 1521-1540.

12. P. Rosa, C. Silvestre, J. Shamma, M. Athans, Fault detection and isolation of LTV systems using set-valued observers, in: Proceedings of the 49th IEEE Conference on Decision and Control, 2010, pp. $768-773$.

13. F. L. Chernousko, Minimax control for a class of linear systems subject to disturbances, Journal of Optimization Theory and Applications 127 (3) (2005) 535-548.

14. A. Kurzhanskiy, P. Varaiya, Ellipsoidal techniques for reachibility analysis of discrete-time linear systems, IEEE Transactions on Automatic Control 52 (1) (2005) 26-38.

15. F. Schweppe, Recursive state estimation: unknown but bounded errors and system inputs, IEEE Transactions on Automatic Control AC-13 (1) (1968) 22-28.

16. I. R. Petersen, A. V. Savkin, Robust Kalman Filtering for Signals and Systems with Large Uncertainties, Birkhauser, New York, NY, USA, 1999.

17. L. Chisci, A. Garulli, A. Vicino, G. Zappa, Block recursive parallelotopic bounding in set membership identification, Automatica 34 (1998) 15-22.

18. E. Kostousova, External polyhedral estimates for reachable sets of linear discrete-time systems with integral bounds on control, Intern. J. Pure and Appl. Math. 50 (2) (2009) 187-194.

19. E. K. Kostousova, State estimation for dynamic systems via parallelotopes optimization and parallel computations, Optimization Methods and Software 9 (4) (1998) 269-306.

20. T. Alamo, J. Bravo, E. Camacho, Guaranteed state estimation by zonotopes, Automatica 41 (2005) $1035-1043$

21. V. Shiryaev, E. Podivilova, Feasible set approximation in dynamic system state guaranteed estimation problem under condition of uncertainty, Mechatronics, Automation, and Control (57) (2014) 10-16.

22. J. Deur, D. Pavković, N. Perić, M. Jansz, D. Hrovat, An electronic throttle control strategy including compensation of friction and limp-home effects, IEEE Trans. Industry Appl. 40 (3) (2004) 821-834.

23. M. di Bernardo, A. di Gaeta, U. Montanaro, J. M. Olm, S. Santini, Experimental validation of the discrete-time MCS adaptive strategy, Control Engineering Practice 21 (6) (2013) 847-859.

24. M. di Bernardo, A. di Gaeta, U.Montanaro, S. Santini, Synthesis and experimental validation of the novel LQ-NEMCSI adaptive strategy on an electronic throttle valve, IEEE Trans. Control Syst. Technol. 18 (6) (2010) 1325-1337.

25. Y. Pan, U. Ozguner, O. H. Dagci, Variable-structure control of electronic throttle valve, IEEE Trans. Industrial Electronics 55 (11) (2008) 3899-3907.

26. A. N. Vargas, L. Acho, G. Pujol, R. C. L. F. Oliveira, J. B. R. do Val, P. L. D. Peres, Robust $H 2$ static output feedback to control an automotive throttle valve, in: Proc. American Control Conf., Portland, Oregon, USA, 2014.

27. X. Jiao, J. Zhang, T. Shen, An adaptive servo control strategy for automotive electronic throttle and experimental validation, IEEE Transactions on Industrial Electronics 61 (11) (2014) 6275-6284.

28. C. Rossi, A. Tilli, A. Tonielli, Robust control of a throttle body for drive by wire operation of automotive engines, IEEE Trans. Control Syst. Technol. 8 (6) (2000) 993-1002.

29. M. Morari, M. Baotic, F. Borrelli, Hybrid systems modeling and control, European Journal of Control 9 (2-3) (2003) 177-189.

30. M. Vasak, M. Baotic, I. Petrovic, N. Peric, Hybrid theory-based time-optimal control of an electronic throttle, IEEE Trans. Industrial Electronics 54 (3) (2007) 1483-1494.

31. X. Yuan, Y. Wang, L. Wu, SVM-based approximate model control for electronic throttle valve, IEEE Trans. Vehicular Technology 57 (5) (2008) 2747-2756. 